\title{
A Comparison of Astronaut Near-Earth Object Missions
}

\author{
Al Globus ${ }^{1}$ \\ San Jose State University, San Jose, CA, 95010 \\ Chris Cassell ${ }^{2}$ \\ Space Studies Institute, Mojave, CA 93501 \\ Stephen Covey ${ }^{3}$ \\ Applied Innovation Inc., St. Augustine, FL, 32092 \\ Jim Luebke L $^{4}$ \\ Space Studies Institute, Mojave, CA 93501 \\ Mark Sonter $^{5}$ \\ Asteroid Enterprises Pty Ltd, Brisbane, Queensland, Australia \\ Bryan Versteeg ${ }^{6}$ \\ Spacehabs.com, Calgary, Alberta, Canada T3H 4W1 \\ and \\ James Wolff \\ Buildatron System, Bethesda, MD 20816
}

September 2012

\begin{abstract}
NASA intends to send astronauts to a near Earth object (NEO) in or around 2025. This is expected to involve a six month mission with a few weeks stay-time at the NEO. Problems with this concept include lack of abort modes, vulnerability to solar flares, and lack of resupply opportunities. Studies by the authors (the Asteroid Mining Group) and a recent workshop at JPL organized by the Keck Institute opens the door to an alternative that addresses these problems and creates additional opportunities. Both groups investigated the feasibility of bringing one of more small NEOs into Earth or Lunar orbit. Particularly for High Earth Orbits (HEO) or High Lunar Orbits (HLO), this appears feasible with near-term technology, especially high-propellant-velocity, low-thrust solar electric propulsion (SEP) inspace vehicles. This paper compares the currently planned mission with an alternative: bringing one or more NEOs into HEO or HLO using SEP and lunar gravity assist. An astronaut mission to the NEO is then similar to a mission to the Moon without a landing. Trip times are measured in days, the NEO can be used for solar flare protection for most of the mission, and resupply within a few days is practical. Furthermore, materials derived from the NEO, e.g., propellant, water, radiation shielding, metals, silicon, and others, are available for projects in cis-lunar space, including satellite refueling, habitats, and space solar power. The alternative mission also develops much of the technology, experience, and infrastructure needed to protect Earth from potentially hazardous NEOs. As an outcome of these studies we are proposing a process whereby early missions can lead to large-scale industrialization of cis-lunar space based on solar energy and asteroidal resources.
\end{abstract}

\footnotetext{
${ }^{1}$ Senior Research Engineer, Research Foundation, 210 N. Fourth St, Fourth Floor, AIAA Member.

21434 Flightline St., AIAA Senior Member.

${ }^{3}$ Director, Research and Development, 1149 Garrison Dr.

${ }^{4} 1434$ Flightline St., AIAA Member.

${ }^{5}$ Consultant, 116 Pennine Dr., South Maclean.

${ }^{6}$ Conceptual Design Visualization Consultant, 117 Wentworth Close SW.

${ }^{7}$ President and Founder, 4803 Westway Dr. 


\section{Nomenclature}

$\begin{array}{ll}A M G & =\text { Asteroid Mining Group } \\ I R & =\text { infra red } \\ I s p & =\text { specific impulse } \\ I S R U & =\text { in situ resource utilization } \\ I S S & =\text { International Space Station } \\ J P L & =\text { Jet Propulsion Laboratory } \\ H E O & =\text { high Earth orbit } \\ H L O & =\text { high Lunar orbit } \\ K I S S & =\text { Keck Institute for Space Studies } \\ L E O & =\text { low Earth orbit } \\ N E O & =\text { near Earth object } \\ P H O & =\text { potentially hazardous object } \\ S E P & =\text { solar electric propulsion } \\ S L S & =\text { space launch system } \\ S S P & =\text { space solar power } \\ \triangle \mathrm{v} & =\text { change in velocity }\end{array}$

\section{Current Plans}

Starting this year, NASA is building a rocket to take astronauts to a NEO. The first version of the Space Launch System (SLS) is expected be the largest rocket since the Saturn V. The budget for the SLS and its companion Orion crew capsule is roughly $\$ 3$ billion per year. The first test flight with people on board is currently scheduled for 20172018. By the time these vehicles make their first operational flight in 2025, America is expecting to pay somewhere around 35 billion dollars for development and testing ${ }^{1}$. Assuming one flight per year for twenty years and one billion per year for operations, each flight will cost around $\$ 3$ billion. Astronaut to NEO missions are not expected every year, so this assumes the SLS will find other suitable customers.

A search of the known NEOs indicates that there is at least one viable target, NEO 1999 AO10, which has a closest approach to Earth in February of 2026, bringing it to within about 10 lunar distances (about four million km). Other targets will undoubtedly be found by search programs as time goes on, indeed some already have been. In any case, a mission to AO10 would probably be timed to rendezvous shortly before its closest approach to Earth, so launch must be near the end of 2025. The entire mission appears feasible within six months with a stay-time of two weeks at AO10. The short stay time is dictated by orbital mechanics. Six months human exposure to weightlessness appears acceptable as this is routine on the International Space Station (ISS). However, as most of the mission will be outside of the Earth's magnetic field, exposure to cosmic rays will be much higher than experienced on the ISS. Also, protecting the crew from a major solar flare will be extremely difficult although the probability of exposure is fairly low. There is no possibility of resupply and for much of the mission abort modes cannot implement a return to Earth in less than a few months.

Activities at the NEO are expected to include direct observation of the surface, taking samples, setting up scientific instruments and testing ISRU (In Situ Resource Utilization, e.g., mining) equipment. Stay times are far too short to process, much less use, significant quantities of local resources, but equipment can be set up and tested. Unfortunately, once the astronauts leave, the ISRU equipment will be of little utility as the NEO will not revisit Earth for many years. For example, AO10s next Earth approach is in 2032 and even then is at a considerably greater distance from Earth.

Thus, NASA's planned mission will involve

1) Six months of transit time.

2) A few weeks stay time at the NEO.

3) Development of a large, powerful, expensive rocket which may have a very limited flight rate.

4) Learning a great deal about one NEO.

5) Providing a step between landing people on the Moon and landing people on Mars.

7) Improved knowledge of NEOs in general, which will help planetary defense. 


\section{Alternate Mission}

In early 2011 a small, informal, after-hours team called the Asteroid Mining Group (AMG), consisting of the authors of this paper, began meetings and email discussions revolving primarily around capturing and returning small asteroids to Earth orbit in accordance with ideas published by group members in $1997^{2}, 1999^{3}$ and at an International Space University project led by an AMG member in 2010. This, and especially, a Keck Institute for Space Studies (KISS) September 2011/January 2012 workshop ${ }^{4}$ held at the Jet Propulsion Laboratory (JPL) opened the door to very different astronaut NEO missions. Based on positive results of an in-house study, the KISS workshop investigated bringing very small NEOs into Earth or Lunar orbit. For NEOs a few meters in diameter this appears feasible. In particular, Damon Landau's presentation at the workshop suggested that a $40 \mathrm{~kW}$ SEP spacecraft expending eight tons of Xenon as reaction mass could bring a 500 ton (with perhaps a 5-10 meter diameter) NEO into High Earth Orbit (HEO). This is possible because the vehicle, which we will call a 'space tug,' uses SEP with a very high propellant velocity and thus uses relatively little propellant. Propellant velocity is around $30 \mathrm{~km} / \mathrm{s}$ (roughly an Isp of $3,000 \mathrm{sec}$ ). Also, a lunar gravity assist supplies up to $1.5 \mathrm{~km} / \mathrm{s} \Delta \mathrm{v}$, depending on how close to the Moon the NEO passes and how rapidly it is moving. Note that the NEOs of interest $(<10 \mathrm{~m}$ diameter $)$ are no threat to Earth.

The feasibility of assisted NEO capture into HEO/HLO is further supported by the observation of one naturally captured NEO, 2006 RH120, a few-meter-diameter object that orbited the Earth for about a year beginning in June of 2006. Recent calculations suggest that on average there is one such object orbiting the Earth at any given time ${ }^{5}$ in very unstable orbits. Adding carefully chosen $\Delta \mathrm{v}$ artificially could increase the population of such objects substantially and place them in stable orbits.

Our ability to bring NEOs into HEO/HLO suggests that with respect to cost, complexity, risk, duration and resupply it may be better to bring NEOs to the astronauts, or at least to places they can easily visit, than send astronauts to a NEO. For these alternate mission the mining equipment stays near Earth, rather than sailing off into the void. That makes products derived from asteroidal materials available for relatively near-term space development. As an added bonus, such missions utilize nearly all the elements of a robust planetary defense: detection of potentially hazardous objects (PHOs -- NEOs with a chance of impacting Earth) and the ability to deflect them.

The NEOs we seek must be small, well under $10 \mathrm{~m}$ diameter, and moving slowly relative to Earth. We have tentatively identified the desirable orbital parameters as $<2.6$ degrees inclination, an eccentricity less than 0.05 (Earth's is 0.0167), and a semi-major axis between 0.92 and $1.15 \mathrm{AU}$. Damon Landau, in his presentation to the Keck workshop ${ }^{1}$, found low-thrust return trajectories for 1991 VG, 2006 RH120, 2007 UN12, and 2008 HU4, all of which seem to fit these criteria or nearly so (some of the eccentricities are a little out of range) with close approaches in the relatively near future. The Keck workshop final report ${ }^{1}$ estimated that current and planned Earth-based telescopes could find roughly five suitable candidates per year with relatively minor additions to current asteroid and comet detection algorithms and procedures. It should be noted that ground telescopes can collect only a small fraction of the light coming from NEOs and therefore have difficulty getting sufficiently accurate orbital parameters and sizes for NEOs, so to enable rendezvous and to upper bound the mass and size observations from radar telescopes are needed.

The total number of suitable NEOs should be very large. To date, approximately 9,000 NEOs have been discovered, at least four of which appear to be suitable. Thus, at a minimum we might expect 0.000444 of all NEOs to be suitable candidates. However, the population of currently known NEOs is statistically skewed toward larger NEOs than those we are interested in. Calculations by Rabinowitz ${ }^{6}$ based on Gehrels ${ }^{7}$ and the orbital parameters criteria above suggest that roughly 0.000765 of all NEOs may meet our criteria, which is in rough agreement. Data from the WISE telescope suggest that there are billions of NEOs at least a few meters in diameter, suggesting that the total number of potential candidates, should they all be found, might be in the millions. Thus, there are sufficient candidates to fuel a large and ongoing flow of material into cis-lunar space. To find large numbers of these, powerful and well placed telescopes would be very helpful.

\section{A. Finding Candidate NEOs}

There is an extensive and growing network of Earth-based telescopes searching the heavens for NEOs. However, this network, being Earth-based, is limited in its capabilities. Specifically, the atmosphere adds noise to the images, the atmosphere blocks much of the infra-red (IR) at the best frequencies for NEO detection, and NEOs on the sunward side of Earth cannot be detected at all. The first two make finding the small NEOs of interest particularly difficult. Furthermore, unlike ground-based optical telescopes, space-based IR telescope can detect most 
frequencies emanating from a NEO. Space telescopes are therefor more likely to gather sufficient data for reasonably accurate size, spin rate, spin axis, and orbital parameters calculations. Thus, a space based telescope or telescopes would be highly desirable.

Harold Reitsema and Robert Aretz proposed a space NEO-detection IR telescope based on existing space telescope designs in a 'Venus-like' orbit $^{8}$, an orbit between Earth and Venus so that NEOs on the sunward side of Earth can be detected. The proposed telescope was designed to find somewhat larger objects, $100 \mathrm{~m}$ across or greater, than we seek and was expected to cost around $\$ 600$ million. They claimed that "this design will detect about $85 \%$ of all $>100$ meter diameter NEOs, about $70 \%$ of all $>60$ meter diameter NEOs, and about $50 \%$ of all $>50$ meter diameter NEOs" during its expected lifetime. The non-profit B612 Foundation has announced plans to launch a cryogenic IR telescope intended to find $90 \%$ of all NEOs greater than $140 \mathrm{~m}$ diameter with a design evolved from the Reitsema-Aretz proposal. The Foundation is negotiating a fixed price contract with Ball Aerospace for telescope manufacture and intends to raise the necessary funds, expected to be a few hundred million dollars, through donations. It should be noted that successful donation-funded projects in the many hundred millions and even billions range are quite common for museums, universities, and so forth.

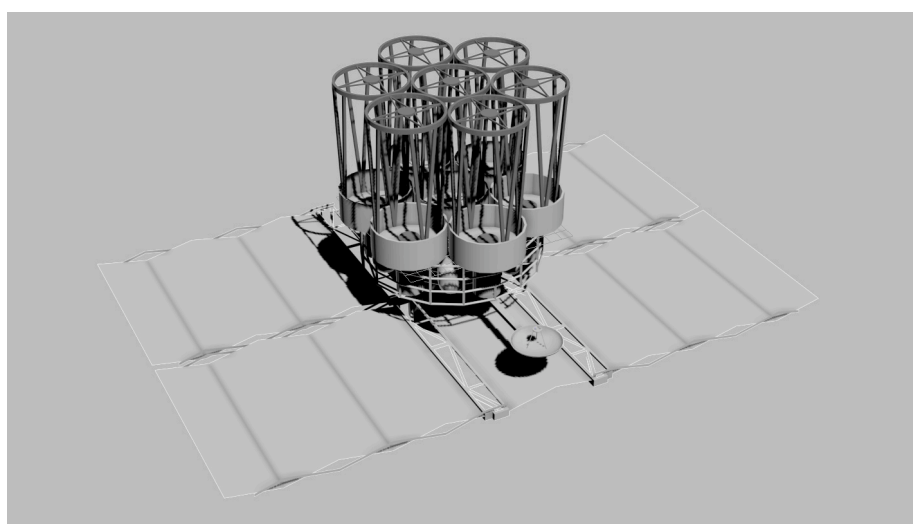

Figure 1: Proposed combined IR/LIDAR telescope sized to fit in a Falcon Heavy faring. Each subscope is $1.5 \mathrm{~m}$ in diameter. The six outside subscopes are ganged together to generate a single image. The center subscope houses a laser that paints the asteroid at the frequency best observed by the outside scopes.

The Asteroid Mining Group is examining a concept for a IR/LIDAR telescope specifically intended for asteroid retrieval activities (see figure 1). Because the objects of interest are small, a larger collection area, six ganged $1.5 \mathrm{~m}$ telescopes in this case, is important. When the six ganged IR telescopes detect a NEO, the central laser system paints the asteroid with an IR laser tuned to the telescope detector's wavelength, increasing the chance of gathering sufficient information to characterize the NEO's size, spin rate, and orbital parameters with sufficient accuracy for rendezvous, capture, despin and return.

In early 2012 a new company, Planetary Resources, Inc., announced that it will pursue asteroid mining backed by a number of very high net worth individuals. Their initial announced activity is the development of a line of small (perhaps nine inch) space telescopes intended to detect and characterize asteroids, among other activities. The idea is to deploy fairly large numbers of the telescopes by driving the price down to a few million dollars per unit with mass production?

If implemented, these telescope systems could perform at least three functions.

1) Detect NEOs for the alternative missions discussed here.

2) Detect NEOs for planetary defense - finding PHOs. The telescopes will be able to find and characterize nearly all of the large objects that cross Earth's orbit and thus give us a nearly complete knowledge of the PHO population.

3) Gather a wealth of data of great scientific interest. In addition to finding and characterizing NEOs, the IR telescopes will find huge numbers of objects in the main belt between Mars and Jupiter. This will give us a near-complete picture of the minor planets in the solar system all the way to Jupiter and perhaps even beyond.

\section{B. Returning NEOs}

The second major piece of necessary hardware is a 'space tug' to apply a small $\Delta \mathrm{v}$ to a NEO to nudge it into a suitable lunar gravity assist. Along these lines, in 1999 Globus et. al proposed the AsterAnts concept ${ }^{3}$. The idea was to capture and return very smalls NEOs $(\sim 1 \mathrm{~m}$ diameter $)$ in large numbers with solar sail powered tugs. This concept did not use a lunar assist as suggested by Mark Sonter in his 1997 asteroid mining thesis ${ }^{2}$ and the $\Delta \mathrm{v}$ required solar sails with a mass similar to the NEO, unless the sail were built in orbit and was thus lighter. Also, 
there was no solution to the despin problem or any way to couple a solar sail to a small NEO. Fortunately, technology has progressed in the intervening years and there is now a viable option that addresses all of these issues.

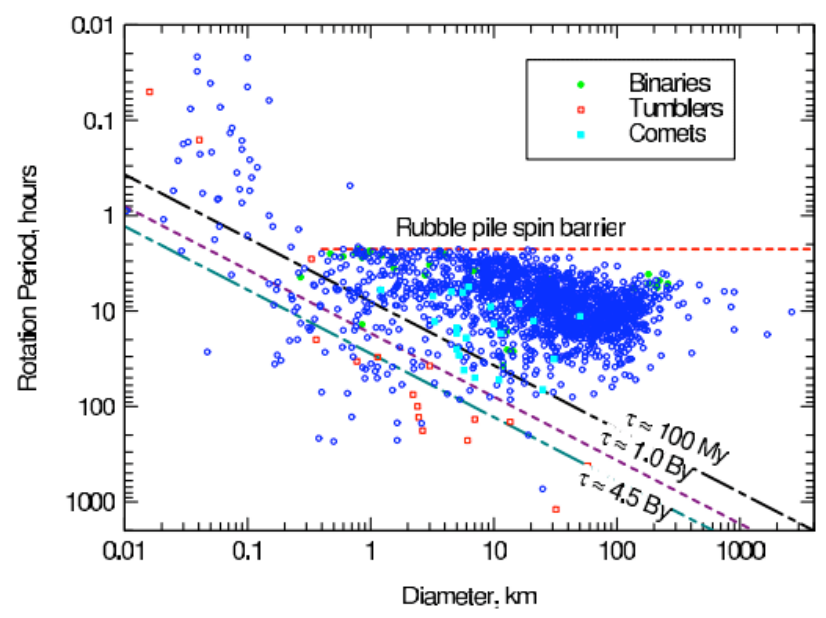

Figure 2: Each dot is an object in free space. The rubble pile spin barrier is the period (approximately 2.2 hours) below which rotational forces will cast loose materials from the equator, thus reducing the angular momentum and rotation rate. Note that almost all objects smaller than 20-30 m diameter have periods below the rubble pile spin barrier and thus must be held together by forces stronger than gravity. Image credit: "Rotational Properties of Asteroids, Comets and TNOs," Harris and Prevec ${ }^{10}$.
Solar electric propulsion (SEP) is the best current option with a flight history due to the very high propellant velocity which minimizes propellant mass for a given $\Delta \mathrm{v}$. Furthermore, applying thrust over long periods of time allows adjustments to the planned thrust program based on observation of the actual dynamics of the system. The Keck Workshop results suggest that 8-12 tons of Xenon propellant is sufficient to return a 500 ton $\mathrm{NEO}$ to $\mathrm{HEO} / \mathrm{HLO}^{1}$ when combined with a lunar gravity assist.

Many large NEOs are known to be rubble piles consisting of many small bodies held together solely by very weak gravitational forces. Applying thrust to a 500 ton rubble pile without breaking the system up would be very challenging and implies that the object must be captured and contained, e.g., by bagging. Fortunately, there is evidence that most small NEOs are solid bodies held together by forces stronger than gravity, see figure $2^{10}$. Note that all of the small objects in figure 2 must be NEOs as more distant small objects cannot be observed by current telescopes. The period of most small NEOs is below the 2.2 hour rubble pile spin barrier and thus they must be solid bodies held together by forces stronger than

gravity. Of course, high spin rates make capture more difficult, but note that there are very small NEOs in figure 2 with a period around one hour. Even higher spin rates can probably be accommodated.

The closest approximation to the tug mission that has been flown is the Japanese Hayabusa mission which landed November 2005 on Itokawa, a NEO a few hundred meters across, and returned a very small sample to Earth in June $2010^{11}$. Like the proposed tug, Hayabusa used SEP but with much less power as a much smaller sample was returned. The spacecraft hovered autonomously to collect samples, demonstrating an important capability, as

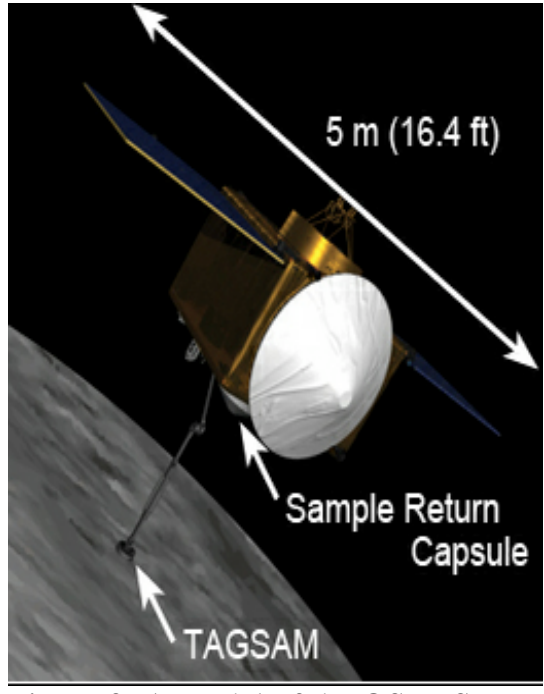

Figure 3: A model of the OSIRIS-Rex sample return spacecraft. The TAGSAM is the sample gathering system. Image credit: University of Arizona rendezvous distances were too great for interactive teleoperation. The sample collected was much smaller than hoped. Furthermore, the spacecraft was seriously damaged and required a heroic engineering effort to bring back to Earth. Hayabusa had a launch mass of around $510 \mathrm{~kg}$ (380 dry). The mission cost around $\$ 170$ million. A follow on mission, Hayabusa 2, is under development with improvements in a number of systems.

NASA and international partners are planning to launch the OSIRIS-REx spacecraft toward 1999 RQ36 to collect and return at least $60 \mathrm{~g}$ of regolith in $2023^{12,13}$. The SEP-powered vehicle, not including the solar arrays, is about $2 \mathrm{~m}$ across. The solar arrays have an area of about $8.5 \mathrm{~m}^{2}$. Total mass is around 1.5 tons. See figure 3 . The spacecraft will not land to acquire the sample but, rather, use a touch-and-go maneuver. This mission is expected to cost around \$1 billion including launch.

The Keck workshop developed a tug design for a mission very similar to what we are proposing here ${ }^{1}$. The Keck tug is a $40 \mathrm{~kW} \mathrm{SEP}$ spacecraft with an inflatable structure for the capture mechanism. The propulsion system consists of five $10 \mathrm{~kW}$ 2-axis gimbaled Hall thrusters with a propellant velocity of around $30 \mathrm{~km} / \mathrm{sec}$. Four of the units are used at any one time with the fifth being an in-flight spare. The launch vehicle is an Atlas V. The design is expected to have a 
mass of around 15.5 tons (including 10 tons of Xenon propellant and $880 \mathrm{~kg}$ of reaction control system propellant) for a total cost of about $\$ 2.6$ billion to return an NEO with a mass of a few hundred tons to HLO. A lunar orbit was chosen to avoid any possibility of dropping the asteroid on Earth.

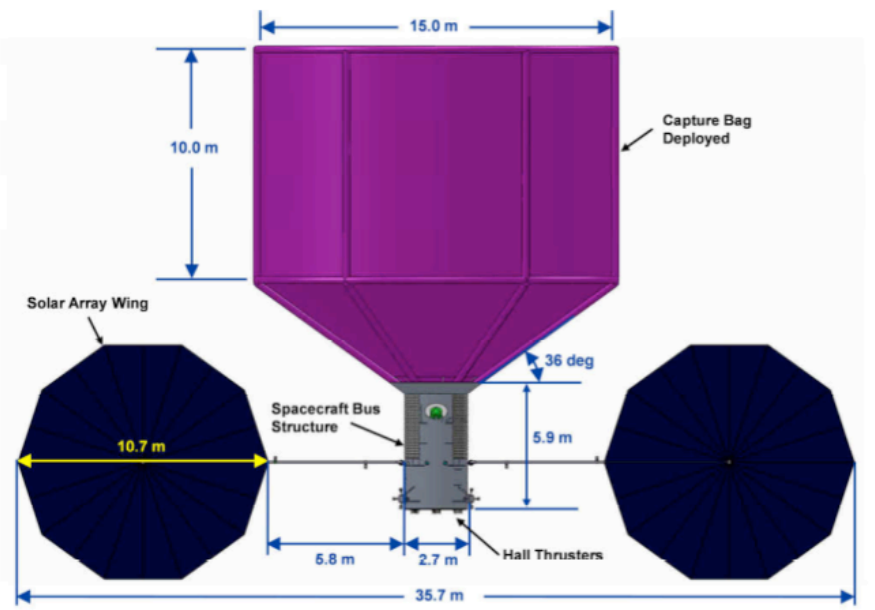

Figure 4: A diagram of the tug proposed by the Keck Workshop. Image credit: Keck Asteroid Retrieval Workshop, COMPASS team at NASA Goddard Research Center.

The Asteroid Mining Group has done some very preliminary investigation into a system similar to the Keck tug, but at (hopefully) lower cost. The idea is to use a communication satellite bus with modifications for deep space operations and NEO capture and retrieval. Comsat SEP systems are expected to be available more-or-less off the shelf at $25 \mathrm{~kW}$ power, using $5 \mathrm{~kW}$ thrusters and a propellant velocity around $18 \mathrm{~km} / \mathrm{sec}$. Rather than use a bag for capture, we are conducting experiments with a simpler and potentially more robust two finger grabber modeled on the PikStick ${ }^{\mathrm{TM}}$ commercial product. The PikStick $^{\mathrm{TM}}$ is used by people to pick up items without bending down and for grabbing items well overhead. The shape and grabbing mechanism are very general purpose and have had extensive terrestrial testing on a wide variety of objects. This is important because the shape and materials property of the target NEO may not be well characterized. Instead of an Atlas V, with a launch cost of about $\$ 200$ million, a Falcon Heavy with a cost around \$100 million is baselined. A complete asteroid mission with this system is expected to cost $\$ 500$ million not including contingency, Deep Space Network charges, or insurance.

Like the telescopes, the space tug can have multiple applications. While designed to return small NEOs to cislunar space, with moderate modifications the same system may also be suitable for small deflections of large NEOs on a collision path with Earth if multiple tugs are employed. A much smaller orbit change is needed for deflection than for a return mission. The PHO just needs be deflected so as to arrive at Earth's orbit a few seconds earlier or later than the Earth itself. Given a large NEO on a collision course with Earth, funding should be plentiful.

Note that if the mission were cancelled at this point humanity would already have benefitted greatly. The location and orbits of nearly all PHOs will be known and the hardware and experience necessary for deflection will have been developed. We will have almost certainly avoided the dinosaurs' fate.

\section{Astronaut Mission}

The first astronaut mission to a NEO brought to cis-lunar space would look much like the mission to an NEO in its natural orbit, with some important differences:

1) Transit time in days, not months.

2) Indefinite stay times, not weeks.

3) Abort to Earth in days, not months.

4) A somewhat smaller launch vehicle should be adequate as the $\Delta v$ is much smaller. For example, the Falcon Heavy, at \$80-125 million per 50 ton-to-LEO launch ${ }^{14}$ (vs billions for the SLS) will probably be adequate. Total additional government development cost: $\$ 0$. SpaceX already has one Falcon Heavy launch contract with Intelsat.

5) When the astronauts return to Earth, the equipment left behind can be used by another mission to the same NEO, or relocated to another NEO.

6) A single astronaut mission can visit multiple NEOs.

7) In case of a solar flare, astronauts can use the NEO for protection for most of the mission. 


\section{Markets}

There is one other major difference. It is at least theoretically possible to develop techniques and infrastructure to the point that mined NEO products can be put up for sale. If so, they will be relatively accessible; not millions of kilometers away. There are a number of potential products, including (in order of difficulty):

1) Samples for scientific study, a proven market.

2) Samples for collectors and museums. There is currently a surprisingly large market for meteorites at prices ranging up to $\$ 1-3,000 / \mathrm{g}$ for samples with known origin and collected shortly after a fall. Pristine asteroidal samples should obtain an even higher price.

3) Radiation shielding. To mimic the atmosphere's protection roughly 10 tons $/ \mathrm{m}^{2}$ is needed. The Bigelow Aerospace BA 330 module has an exterior surface area of about $365 \mathrm{~m}^{2}$. To provide a radiation protected space would thus need approximately 3,650 tons of material - or seven 500 ton NEOs.

4) Water. Water is needed for life support and can be split into $\mathrm{H}_{2}$ and $\mathrm{O}_{2}$ for rocket fuel and oxidizer. Unlike radiation shielding, to produce water from NEOs requires substantial processing.

5) Metals. Most NEOs contain some metal and many contain a great deal. There are many potential markets, but the largest is for space solar power (SSP) satellites. While economical SSP will be difficult to develop, the potential payoff is huge: vast quantities of clean energy for millions of years. Supplying the metals from NEOs could radically reduce the mass launched from Earth and eliminate most of the environmental impact of producing SSP space-segment components on the ground and launching them. Although upfront SSP costs are increased, there is evidence that break-even on investment is earlier ${ }^{15}$ using extraterrestrial materials.

6) Silicon. Nearly all NEOs have ample quantities of silicon. This could also be useful for SSP.

7) High value metals. Some NEOs contain large quantities of extremely valuable materials such as platinum group metals (PGMs). At some point, it may be possible to supply Earth markets with these.

Note that 1,2, and 7 require delivering materials to Earth, whereas 3-7 are in-space delivery.

This approach to NEO missions promises at least the possibility of relatively near-term development of industrial infrastructure in cis-lunar space. NEOs are not just objects of study, not just threats to be dealt with, but the raw materials for space industrialization on a large scale.

It should be noted that once there are NEOs in cis-lunar space, setting up mining operations will very likely require human presence. On Earth a number of mines are teleoperated, with no personnel in the mine at all. These operations are conducted in the presence of considerable (9-10 sec) delay, comparable to what one might expect from speed-of-light and information processing delays for teleoperating objects in near-lunar orbits from Earth. Although terrestrial mines are sometimes teleoperated, none have been set up without human workmen nor is this likely in the foreseeable future; there are too many unknowns. Thus, the astronaut missions are an important part of setting up NEO mining operations to serve existing and future markets.

\section{Comparison}

The currently planned astronaut mission to a NEO is just that, a mission. The alternative presented here is not just a mission, it is a pathway to space industrialization. To summarize the difference between sending astronauts to explore far off NEOs vs. bringing small NEOs into cis-lunar space for human exploration and exploitation, consider table 1 . 


\begin{tabular}{|l|l|}
\hline Current NASA Mission Concept & Alternate Mission Concept: Industrialization \\
\hline Six months travel time & Six days travel time \\
\hline No rapid return & Return in three days \\
\hline No resupply & Resupply in three days \\
\hline Fixed, short stay times & Indefinite stay time \\
\hline Large $\Delta \mathrm{v}$, expensive new vehicle & Smaller $\Delta \mathrm{v}$ for astronauts, Falcon Heavy sufficient \\
\hline One NEO per mission & Potentially many NEOs per astronaut flight \\
\hline Repeat visits to same NEO difficult & Repeat visits easy \\
\hline Only supply NEO materials science market & Potentially supply multiple NEO materials markets \\
\hline Some contribution to planetary defense & Includes full planetary defense system (detection and deflection) \\
\hline
\end{tabular}

Table 1: Comparison of missions.

Thus, when considering an astronaut mission to a NEO, we should carefully consider what our real goals are. If our goal is bragging rights for sending astronauts to a large object very far away, then the current mission concept works well. If our goal is to extend commerce, industry, humankind and Life itself into space, then the alternative presented here, or something similar, should be carefully considered.

\section{Conclusion}

While NASA's current plans include sending astronauts to a NEO far beyond the Earth, this may not be the best approach to exploiting NEOs. In particular, most or all of the knowledge to be gained could be found by bringing one or more NEOs into cis-lunar space for astronaut exploration. The human part of such a mission or set of missions is much easier and safer than a venture far out into the void. Perhaps more importantly, the importation of large quantities of materials into orbit within the Earth-Moon system and the ability to bring more could lead to creation of a large, commercially-viable NEO mining industry with incalculable positive impact on human development.

\section{Acknowledgements}

We would like to thank the Space Studies Institute for organizational support.

\section{References}

1. Covault, C. SLS Development Begins. Aerospace America 41-45 (2012).

2. Sonter, M. The Technical and Economic Feasibility of Mining the Near-Earth Asteroids. Masters of Science Thesis (Honors), University of Wollongong, Department of Physics and Department of Civil and Mining Engineering (1997) at <http://www.nss.org/settlement/asteroids/MiningNearEarthAsteroids(Sonter).pdf August 2012>

3. Globus, A., Biegel, B. \& Traugott, S. AsterAnts: A Concept for Large-Scale Meteoroid Return and Processing. NASA Ames NAS technical report NAS-99-006. Presented at Space Frontier Conference 8 (1999) at $<$ http://alglobus.net/NASAwork/papers/AsterAnts/paper.html August 2012>

4. Brophy, J. et al. Asteroid Retrieval Feasibility Study. (Keck Institute for Space Studies: 2012) at $<$ http://kiss.caltech.edu/study/asteroid/asteroid_final_report.pdf $>$

5. Granvik, M., Vaubaillon, J. \& Jedicke, R. The Population of Natural Earth Satellites. Icarus (2012).doi:10.1016/j.icarus.2011.12.003

6. Rabinowitz, D. L. Asteroid Distribution Question personal communication. (2012).

7. Gehrels, T. Hazards Due to Comets and Asteroids. (University of Arizona Press: 1994).

8. Reitsema, H. \& Arentz, R. NEO Survey: An Efficient Search for Near-Earth Objects by an IR Observatory in a Venus-like Orbit. (Primitive Bodies Subcommittee of the Decadal Survey) at $<$ http://www.lpi.usra.edu/decadal/sbag/topical_wp/RobertFArentz.pdf August 2012>

9. Mann, A. Tech Billionaires Plan Audacious Mission to Mine Asteroids. Wired (2012) at <http://www.wired.com/wiredscience/2012/04/planetary-resources-asteroid-mining August 2012> 
10. Harris, A. \& Pravec, P. Rotational Properties of Asteroids, Comets and TNOs. Asteroids, Comets, Meteors Proceedings IAU Symposium 229, (2005).

11.Kuninaka, H. \& Kawaguchi, J. Lessons Learned from Round Trip of Hayabusa Asteroid Explorer in Deep Space. $1-8(2011)$ at

$<\mathrm{http}: / /$ ieeexplore.ieee.org/xpl/login.jsp?tp=\&arnumber=5747599\&url=http\%3A\%2F\%2Fieeexplore.iee.. org\%2 Fxpls\%2Fabs_all.jsp\%3Farnumber\%3D5747599 August 2012>

12. Origins Spectral Interpretation Resource Identification Security Regolith Explorer Fact Sheet at <http://www.nasa.gov/centers/goddard/pdf/552572main_OSIRIS_REx_Factsheet.pdf August 2012>

13. OSIRIS-REx Web Site. at <http://osiris-rex.lpl.arizona.edu/ August 2012>

14. SpaceX Falcon Heavy web page. (2012).at <http://www.spacex.com/falcon_heavy.php August 2012>

15.Curreri, P. A. \& Detweiler, M., K. A Contemporary Analysis of the O'Neill - Glaser Model for Space-based Solar Power and Habitat Construction. NSS Space Settlement Journal (2011) at $<$ http://www.nss.org/settlement/journal/NSSJOURNAL_AnalysisOfONeill-GlaserModel_2011.pdf August 2012>

Copyright $\odot 2012$ by Al Globus. Published by the American Institute of Aeronautics and Astronautics, Inc., with permission 